\title{
Chebulinic Acid Negated the Development of Streptozotocin- Induced Experimental Dementia in Rats
}

\author{
Arora Rimpi ${ }^{1,2}$, Deshmukh Rahul ${ }^{3 *}$ \\ 'Research Scholar, I.K. Gujral Punjab Technical University, Jalandhar, Punjab, INDIA. \\ ${ }^{2}$ Neuropharmacology Division, Department of Pharmacology, I. S. F. College of Pharmacy, Moga, Punjab, INDIA. \\ ${ }^{3}$ Department of Pharmaceutical Sciences and Technology, Maharaja Ranjit Singh Punjab Technical University, Bathinda, Punjab, INDIA.
}

\begin{abstract}
Background: With the constant failure of the clinical trials and continuous exploration of a therapeutic target against Alzheimer's disease (AD) is the utmost needed. Chebulinic acid (ChA) has been reported to possess neuroprotective potential in various neurodegenerative models such as anxiety and depression. Materials and Methods: In the current study, the ChA was challenged on the progression of $A D$ induced by intracerebroventricular (ICV)-streptozotocin (STZ)-induced neurotoxicity to determine its therapeutic potential in experimental dementia. STZ was infused bilaterally ( 3 $\mathrm{mg} / \mathrm{kg} / \mathrm{icv}$ ) on day $1^{\text {st }}$ and $3^{\text {rd }}$ after surgery. ChA $(25,50$ and $100 \mathrm{mg} / \mathrm{kg} / \mathrm{p} .0)$ was administered from $7^{\text {th }}$ day onwards up to $21^{\text {st }}$ day following $1^{\text {st }}$ ICV-STZ infusion. Cognitive impairment was evaluated by actophotometer, Morris water maze (MWM) and object recognition task (ORT) in rats whereas biochemical, neurochemical, neuroinflammatory were evaluated using hipoocampal brain regions on day $22^{\text {nd }}$. Results: Ventricular administration of STZ in rats found to significantly shorten the latency time on the MWM and ORT which was associated with significant alterations in hippocampal biochemistry, including elevation in oxidative stress and compromised
\end{abstract}

antioxidant defense, neurotransmitter alteration and elevation in neuroinflammatory cytokine levels. ChA treatment significantly prevented the ICVSTZ-induced memory deficit by attenuating the hippocampal neuronal loss, neuroinflammation and compromised antioxidant defense and cholinergic deficits in rats. Conclusion: These results clearly pointed to the pivotal role of ChA in ICV-STZ induced neurotoxicity and its association may be a promising alternative to be investigated in the treatment of AD-like dementia. Key words: Alzheimer's Dementia, Chebulinic acid, Streptozotocin, Neuroprotection, Hippocampus.

\section{Correspondence}

Dr. Rahul Deshmukh, M. Pharm.,

Department of Pharmaceutical Sciences and Technology, Maharaja Ranjit Singh Punjab Technical University, Bathinda-151001, Punjab, INDIA.

Phone no: +919877039519

Email: drrahuld09@gmail.com

DOI: 10.5530/ijpi.2020.1.5

\section{INTRODUCTION}

A disease of unknown etiology, Alzheimer's disease (AD) is the most common type of dementia without care ${ }^{1}$ and most $\mathrm{AD}$ cases are sporadic where age represents the greatest risk factor. ${ }^{2}$ According to the World Alzheimer Report 2018, there are currently about 46.8 million people suffering with $\mathrm{AD}$ worldwide. ${ }^{3}$ The ageing of world population will further compound this problem and lead to a steep increase in the number of $\mathrm{AD}$ patients. ${ }^{4}$ Causes of $\mathrm{AD}$ are not yet fully understood but advances in brain imaging have allowed researchers to see the development and accumulation of extracellular amyloid beta $(\mathrm{A} \beta)$ plaques and intraneuronal neurofibrillary tangles, in discrete regions of the basal forebrain, association cortices; including hippocampus (part of temporal lobe of the brain responsible for processing of memory), as well as shrinkage in brain structure and change in its function. ${ }^{5}$ These affects worsens with age and consequently leads to atrophy (shrinking) of certain parts of the brain, oxidative stress, neuroinflammation, mitochondrial dysfunction, cholinergic deficits and gliosis along with dystrophic neurites, loss of neurons and synapses, accompanied by psychological and pathophysiological complications such as anxiety, depression, concentration problems and motor disturbances. ${ }^{2,6}$ Numerous in vivo studies have demonstrated that Insulin resistance, oxidative stress, glutamate excitotoxicity, mitochondrial dysfunction and neuroinflammation are also among the major pathophysiological features of $\mathrm{AD} .^{7,6}$ The intracerebroventricular (ICV) injection of streptozotocin results in a well-established rat model showing many aspects of SAD including neuroinflammation, brain insulin resistance, cholinergic deficits, accumulation of $\beta$-amyloid and tau proteins and oxidative stress as well as memory and learning impairment. ${ }^{8,9}$
At present, the disorder is not curable; because available therapies help to maintain neuronal function, they do not provide a significant impact on the reversal of the disease process. ${ }^{10,11}$ Based on these facts, the employment of natural products with infinitesimally, noticeable side effects constitute substitutes for treating these neurodegeneration. The plant Terminalia chebula also known as Haritaki has an esteemed origin in Indian mythology. Terminalia chebula is a deciduous tree growing up to 30 -metre ( $98 \mathrm{ft}$ ) tall, with a trunk up to 1 -metre $(3 \mathrm{ft} 3 \mathrm{in}$ ) in diameter. The fruits are drupe-like, long, broad and blackish, with five longitudinal ridges and are hard and yellowish-green in colour. ${ }^{12}$ The plant contain diverse chemical constituents such as ellagic acid, gallic acid, ellagitannins and gallotannins ${ }^{13}$ and have been known for a long time to show pharmacological effects like cytoprotective, ${ }^{14}$ antidiabetic, ${ }^{15}$ antioxidant ${ }^{16}$ antiarthiritic. ${ }^{17}$ It has been reported that Terminella chebulla also posses acetyl cholinesterase inhibitory ${ }^{18}$ and neuroprotective potential ${ }^{19,20}$ e.t.c. Chebulinic acid (ChA) is an ellagitannin found in the fruits of Terminalia chebula. It has the molecular formula of $\mathrm{C}_{41} \mathrm{H}_{32} \mathrm{O}_{27}$ and molecular weight of $956.67658[\mathrm{~g} / \mathrm{mol}] .{ }^{12}$ Other studies have shown that chebulinic acid has reported to posses unique biochemical and pharmacological properties such as antidiabetic, ${ }^{21}$ antimutagenic, ${ }^{22}$ anti-apoptotic, antioxidant, ${ }^{23,10}$ anti-inflammatory, ${ }^{17}$ ischaemic reperfusion injury, ${ }^{24}$ actylcholinesterase inhibitory and free radical scvanging activity ${ }^{18,25}$ and cardio and hepatoprotective effects. ${ }^{16}$ Moreover, ChA has been reported to show anxiolytic, antidepressant ${ }^{12}$ and protective potential in glutamate induced cell death experimental animals. ${ }^{26}$ However, there has not been any report of the antidementic activity of chebulinic acid. Therefore, the

This is an open access article distributed under the terms of the Creative Commons Attribution-NonCommercial-ShareAlike 4.0 License, which allows others to remix, tweak, and build upon the work non-commercially, as long as the author is credited and the new creations are licensed under the identical terms. 
objective of this present study is to evaluate the antidementia potentials of ChA in laboratory rats using universally accepted experimental model: ICV-STZ model. Herewith, the current study may prove the use of $\mathrm{ChA}$ as therapeutic approach in amelioration and/or delaying the detrimental effects of AD.

\section{MATERIALS AND METHODS}

\section{Animals}

Adult male Wistar rats, weighing 250-280 g were procured from Lala Lajpat Rai University of Veterinary and Animal Sciences, Hisar and kept in Central Animal House of ISF College of Pharmacy, Moga, Punjab (India). The animals were housed in polyacrylic cages in a well- controlled atmosphere (room temperature $22 \pm 2^{\circ} \mathrm{C}$ and relative humidity of $60 \%$ ) with $12 \mathrm{hr}$ light/dark cycle (lights sturned on at $7 \mathrm{AM}$ ). The animals were maintained on a commercial diet in the form of dry pellets and water ad libium. All the behavioral parameters were assessed between 9:00 and 17:00 hr. The protocol of the study was approved by the Institutional Animal Ethics Committee (IAEC) and was carried out in accordance with the Committee for the Purpose of Control and Supervision on Experiments on Animals (CPCSEA) guidelines for the use and care of experimental animals. All the experiments for a given treatment were performed using age-matched animals in an effort to avoid variability between experimental groups.

\section{Drugs and Chemicals}

Streptozotocin (STZ) and acetylthiocholine iodide (AChI) 5, 5'-dithiobis (2-nitrobenzoic acid) (DTNB) and ChA were purchased from SigmaAldrich, USA. STZ was diluted in citrate buffer ( $\mathrm{pH} 4.4)$ and ChA was always prepared afresh by dissolving in 1\% CMC (Carboxymethylcellulose). Interleukin-1 beta (IL-1 $\beta$ ), Interleukin-6 (IL-6) and Tumor necrosis factor-alpha (TNF- $\alpha$ ) Elisa Kits were purchased from Krishgen Biosystem, India. Unless stated, all other chemicals and biochemical reagents of highest analytical grade were used for the study. Solutions of the drugs and chemicals were freshly prepared before use.

\section{Intracerebroventricular infusion of streptozotocin}

Rats were anaesthetized with thiopentone sodium (35 mg/kg, i.p) and xylazine $(5 \mathrm{mg} / \mathrm{kg}$, i.p). The head was placed in position in the stereotaxic apparatus (Stoelting Co. USA, Model no: 53311). Briefly, a midline saggital incision was made in the scalp. Two holes were drilled through the skull and the infusion cannula was placed into the lateral cerebral ventricles (coordinates: $0.8 \mathrm{~mm}$ posterior to bregma; $1.5 \mathrm{~mm}$ lateral to saggital suture; $3.6 \mathrm{~mm}$ ventral from the surface of the brain ). ${ }^{27}$ Following cannulae placement animals were injected with gentamicin $(5 \mathrm{mg} /$ $\mathrm{kg}$ ) and were placed in individual cages. Animals were observed for one week and special care was taken by administering sweetened milk daily, during the resting phase for recovery (Figure 1). Streptozotocin was dissolved in citrate buffer ( $\mathrm{pH} 4.4)$ just prior to administration and slowly injected $(1 \mu \mathrm{l} / \mathrm{min}$ ) (infusion pump QSI 53311) through the cannula using Hamilton microsyringe in a volume of $10 \mu$ into each lateral cerebral ventricle (bilateral ICV) on day 1 and 3 as described previously. ${ }^{28}$

\section{Experimental groups}

Animals were divided into five groups and each group comprised of 10 animals. The treatment schedule and the interval for estimation of various parameters are presented in Figure 1. Group 1: served as sham control; Group 2: Rats were infused with ICV-STZ $(3 \mathrm{mg} / \mathrm{kg} / 10 \mu \mathrm{l})$ with infusion rate $1 \mu \mathrm{l} / \mathrm{min}$ into each cerebral ventricle (bilateral ICV). Group 3, 4 and 5: received Chebulinic acid at doses of 25,50 and $100 \mathrm{mg} / \mathrm{kg}$,

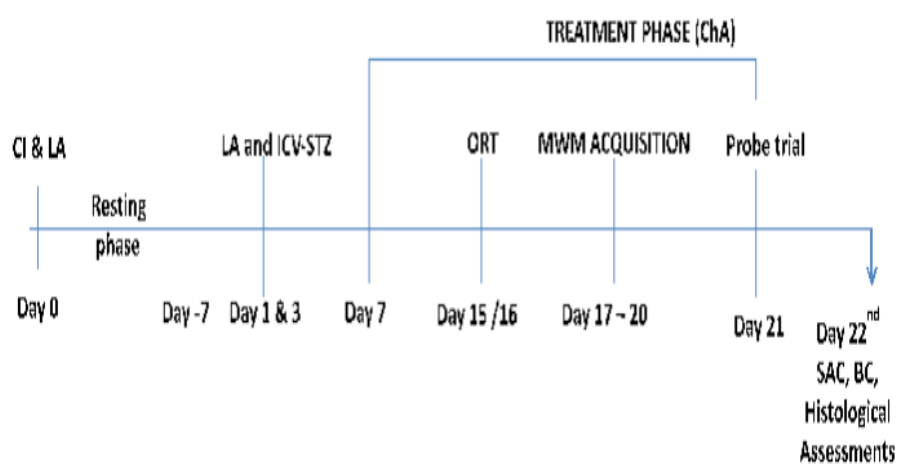

Figure 1: Experiment Procedure and Treatment Schedule.

p.o., respectively, starting from day 7 after the $1^{\text {st }}$ dose of STZ infusion and continued once daily for a period of 21 days.

\section{Behavioral assessment}

\section{Spontaneous locomotor activity}

Each animal was tested for spontaneous locomotor activity on day $22^{\text {nd }}$ following $1^{\text {st }}$ ICV-STZ infusion. Each animal was observed over a period of $10 \mathrm{~min}$ in a square closed arena equipped with infrared light sensitive photocells using a digital photoactometer (INCO, India). ${ }^{28}$

\section{Morris water maze test}

Spatial learning and memory of animals in Morris water maze (MWM) was tested by the method described. ${ }^{29}$

\section{Object Recognition task (ORT)}

Novel Object recognition (NOR) test was performed for analyzing nonspatial and short-term memory in rats. We followed the protocol previously described by ${ }^{30}$ with minor modifications according to. ${ }^{29}$

\section{Biochemical Assessments}

On $22^{\text {nd }}$ day, after completion of behavioral analysis, rats were sacrificed under light ether anesthesia. Blood was completely removed from the brain tissues using perfusion technique with phosphate buffer through the heart to avoid any interference with the homogenate readouts. The brain was carefully removed from the skull and rinsed with ice-cold isotonic saline. Hippocampal tissues were separated from the whole brain and then homogenized $\{10 \%(\mathrm{w} / \mathrm{v})\}$ in ice-cold phosphate buffer $(0.1 \mathrm{M}$; $\mathrm{pH} 7.4)$ at $10,000 \mathrm{~g}$ for $15 \mathrm{~min}$ at $\left(4^{\circ} \mathrm{C}\right)$. Supernatants were separated and stored at $-80^{\circ} \mathrm{C}$ for performing biochemical estimations. Hippocampal Protein was measured by the method of ${ }^{31}$ using bovine serum albumin $(1 \mathrm{mg} / \mathrm{ml})$ as a standard.

\section{Acetylcholinesterase (AChE) assay}

The quantitative measurement of AChE activity in brain hippocampus was performed according to the method described by Ellman et al. ${ }^{32}$

\section{Estimation of malondialdehyde (MDA)}

The quantitative measurement of MDA end product of lipid peroxidation in brain hippocampus homogenate was performed according to the method of Wills. ${ }^{33}$

\section{Estimation of reduced glutathione (GSH)}

Reduced glutathione in brain hippocampus was estimated according to the method described by Ellman. ${ }^{34}$ 


\section{Estimation of nitrite}

The accumulation of nitrite in the hippocampus supernatant, an indicator of the production of nitric oxide (NO), was determined by a colorimetric assay using Greiss reagent $(0.1 \% \mathrm{~N}$-(1-naphthyl) ethylenediamine dihydrochloride, $1 \%$ sulfanilamide and $2.5 \%$ phosphoric acid) as described by Green et al. ${ }^{35}$

\section{Neurochemical estimation}

\section{Estimation of catecholamines}

Catecholamines, dopamine, 5 hydroxytryptymine and norepinephrine (DA, 5-HT and NE) levels were estimated by HPLC using electrochemical detector according to the method described by Arora and Deshmukh. ${ }^{29}$

\section{Estimation of GABA and glutamate}

The estimation of GABA and glutamate was done by method described by Donzanti and Yamamoto ${ }^{36}$ with slight modifications as described by Arora and Deshmukh. ${ }^{29}$ The values are expressed as percentage of Normal Control group.

\section{Estimation of pro-inflammatory cytokines (IL-1 $\beta$, IL-6 and TNF-a) levels}

The quantifications of IL- $1 \beta$, IL- 6 and TNF- $\alpha$ were done by using rat IL$1 \beta$, IL- 6 and TNF- $\alpha$ immunoassay kit (Krishgen Biosystems, India). The quantikine rat IL- $1 \beta$, IL-6and TNF- $\alpha$ immunoassay is a $4.5 \mathrm{~h}$ solid phase ELISA designed to measure IL- $1 \beta$, IL- 6 and TNF- $\alpha$ levels. It is a solidphase sandwich enzyme linked immunosorbent assay (ELISA) using a microtitreplate reader. Concentrations of proinflammatory cytokines were calculated from the standard curves.

\section{Statistical analysis}

The results were analyzed using Graph Pad Prism 6.01 (San Diego, CA, United States) and values were expressed as mean \pm standard error mean (SEM). Escape latency period in MWM and total exploration time in T1, T2 on familiar and novel object, in NOR was measured using two way analysis of variance (ANOVA). Catecholamines, GABA and glutamate and proinflammatory were analyzed by repeated measure two way ANOVA followed by Bonferroni's post hoc test for multiple comparisons and others behavior and biochemical parameters were analyzed by one way ANOVA followed by Tukey's post-hoc test. Values with $P<0.05$ and $P<0.001$ were considered to be statistically significant.

\section{RESULTS}

\section{Effect of ChA on spontaneous locomotor activity in ICV- STZ infused rats}

The spontaneous locomotor activity on Day 0 , Day 1 and $22^{\text {nd }}$ did not differ significantly among all the groups $(p>0.001)$ (Table 1$)$, suggesting no effects whatsoever of ChA (25, 50 and $100 \mathrm{mg})$ or STZ on this parameter in the current study.

\section{ChA attenuated ICV-STZ induced memory deficit during Morris Water Maze (MWM) task in rats.}

Repeated measure two way ANOVA analysis indicated overall significant effect of treatment, time and a time $\times$ treatment interaction $(p<0.001)$. The latencies to reach the submerged platform decreased gradually in all the groups during 4 days of training in Morris water maze (MWM) task (Figure 2a), except those of the ICV STZ infused group of animals, day 17 to $20(p<0.001)$ as compared with those of sham control, indicating poorer learning abilities following STZ administration. Chronic administration of ChA dose dependently attenuated STZ induced acquisition deficit $(P<0.001)$. ChA treated rats showed improved learning abilities as compared to STZ control rats.

During the probe trial, with the platform removed, STZ infused rats failed to remember the precise location of the platform, spent less time in the target quadrant as compared with sham control animals $(p<0.001$, Figure $2 \mathrm{~b}$ ). On the hand ChA treated rats were able to locate the target quadrant and \% time spent in target quadrant was significantly higher to that of STZ control rats indicating improved consolidation of memory ( $p$ $<0.001$, Figure 2b).

\section{ChA reverses ICV-STZ induced impairment in short term} recognition memory task performance in rats.

Non-spatial memory and Short-term memory was assessed using Novel Object Recognition (NOR) test. On day 15 following ICV-STZ infusion,

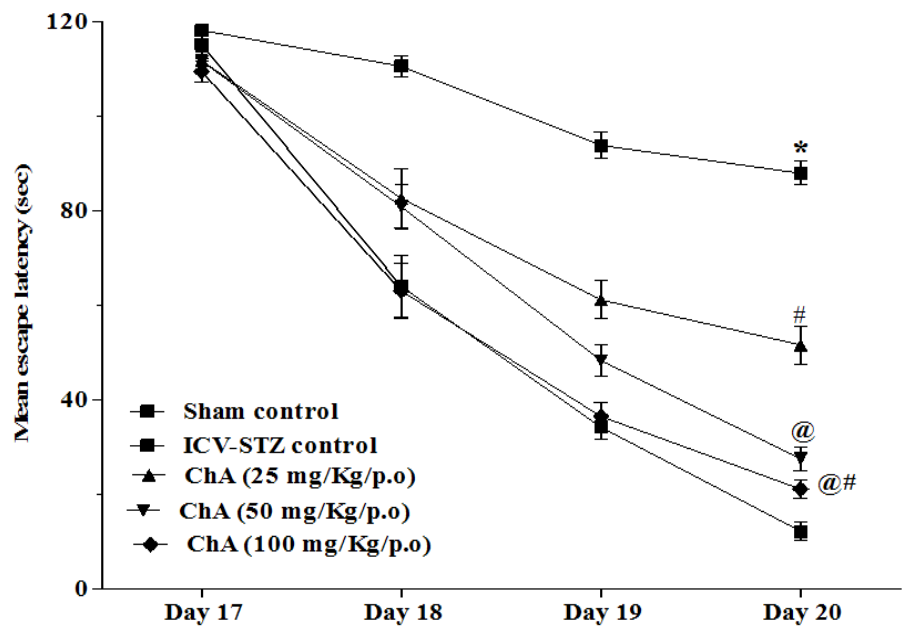

Figure 2a: Effect of ChA on memory performance (mean escape latency) in Morris water maze task in intracerebroventricular streptozocin (ICV-STZ) treated rats.

Values are expressed as mean $\pm \mathrm{SEM},{ }^{*} P<0.001$ vs Sham, $\# P<0.001$ vs STZ, @ $P<0.001$ vs ChA (25mg/kg), @\# $P<0.001$ vs ChA (50mg/kg)

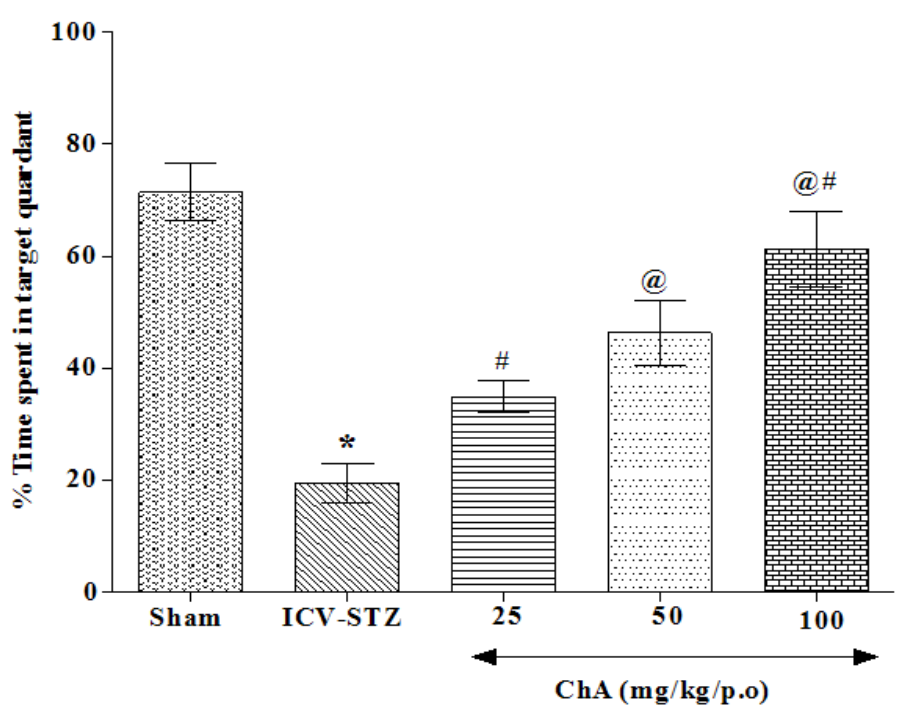

Figure 2b: Effect of ChA on time spent in target quadrant intracerebroventricular streptozocin (ICV-STZ) treated rats.

Values are expressed as mean $\pm \mathrm{SEM},{ }^{*} P<0.001$ vs Sham, $\# P<0.001$ vs STZ, @ $P<0.001$ vs ChA ( $25 \mathrm{mg} / \mathrm{kg})$, @\# $P<0.001$ vs ChA (50mg/kg) 
during the first test (T1), the non-significant difference was observed during familiarize phase in between all treatment groups $(\mathrm{p}>0.001)$ (Figure 3a). On the second day (16, T2), two way ANOVA analysis indicated overall significant discrimination effect of treatment, when animals were exposed with familial (FO) and novel object (NO), STZinfused rats were not able to discriminate them and spent equal time to explore the FO and NO. Whereas, treatment with ChA (25, 50 and 100 $\mathrm{mg} / \mathrm{kg}$ p.o) significantly and dose dependently improved STZ-induced object discriminative ability in animals and the animals spent more time on, when exposed to FO and NO $(P<0.001$, Figure $3 \mathrm{~b})$. ChA $(100 \mathrm{mg} / \mathrm{kg}$ p.o) exhibit maximum effect amongst various doses tried (Figure $3 \mathrm{a}$ and $3 \mathrm{~b})$. Moreover, one way ANOVA with post- hoc comparisons showed significant difference in discrimination index (DI), there was significant

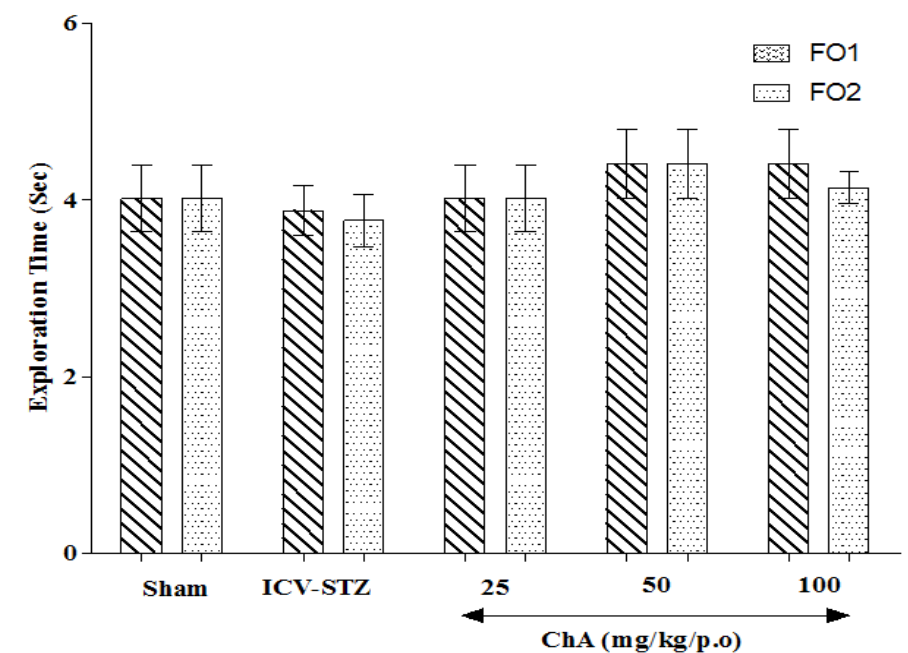

Figure 3a: Effect of ChA on memory performance (acquisition phase, Day $15^{\text {th }}$ ) in object recognition test in intracerebroventricular streptozocin (ICVSTZ)- treated rats.

Values are expressed as mean \pm SEM, ${ }^{*} P<0.001$ vs Sham, $\# P<0.001$ vs STZ, @ $P<0.001$ vs ChA (25mg/kg), @\# $P<0.001$ vs ChA (50mg/kg).

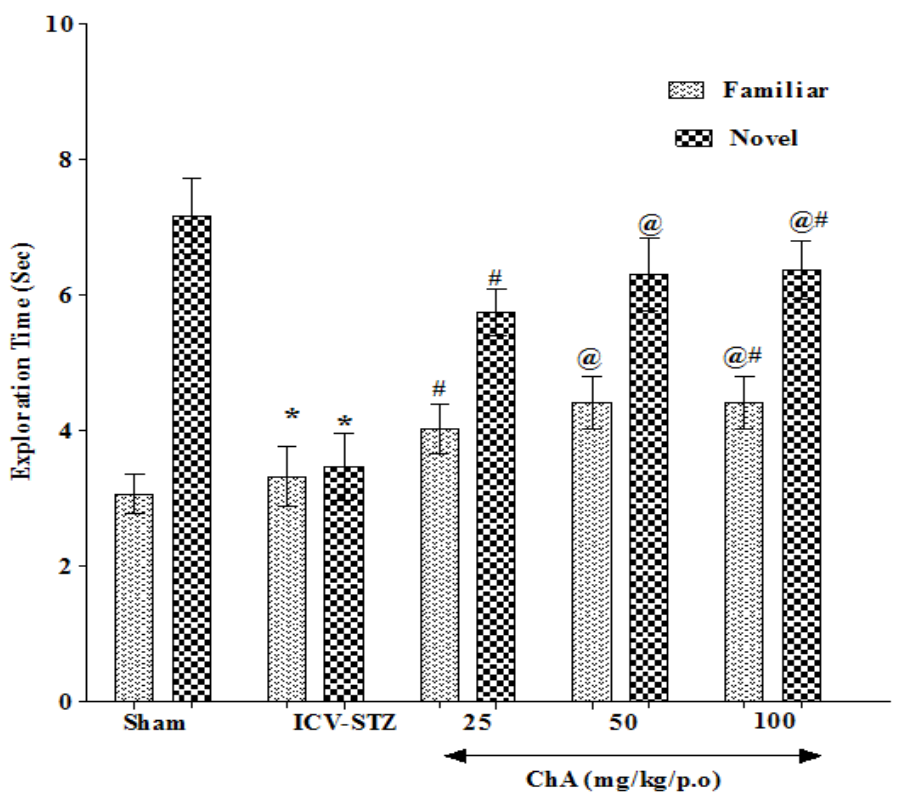

Figure 3b: Effect of ChA on memory performance (Retention phase, Day $16^{\text {th }}$ ) in object recognition test (ORT) in intracerebroventricular streptozocin (ICV-STZ)- treated rats.

Values are expressed as mean $\pm \mathrm{SEM},{ }^{*} P<0.001$ vs Sham, $\# P<0.001$ vs STZ, @ $P<0.001$ vs ChA (25mg/kg), @\# $P<0.001$ vs ChA (50mg/kg).

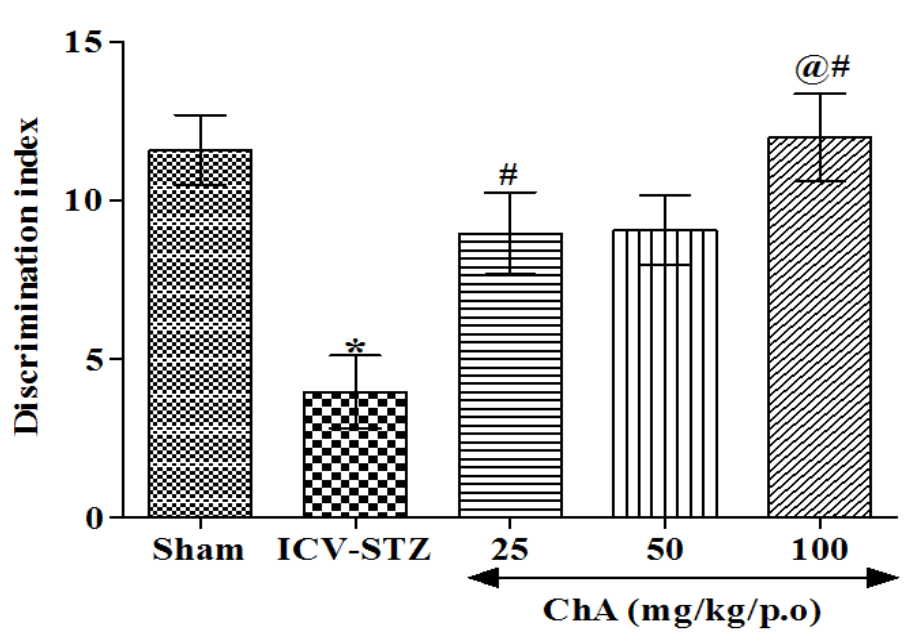

Figure 3c: Effect of ChA on memory performance (Discrimination index, Day $16^{\text {th }}$ ) in object recognition test (ORT) in intracerebroventricular streptozocin (ICV-STZ)- treated rats.

Values are expressed as mean $\pm \mathrm{SEM},{ }^{*} P<0.001$ vs Sham, $\# P<0.001$ vs STZ, $@ P<0.001$ vs ChA ( $25 \mathrm{mg} / \mathrm{kg})$, @\# $P<0.001$ vs ChA (50mg/kg)

Table 1: Effect of ChA on brain (hippocampus) catecholamines level in intracerebroventricular streptozocin (ICV-STZ) treated rats.

\begin{tabular}{|c|c|c|c|}
\hline \multirow[t]{2}{*}{ Groups } & \multicolumn{3}{|c|}{$\begin{array}{c}\text { Biochemical Parameters (pg/ml) } \\
\mathrm{ng} / \mathrm{mg} \text { tissue sample (\% of control) }\end{array}$} \\
\hline & Norepinephrine & Dopamine & Serotonin \\
\hline Sham control & $91.875 \pm 6.717$ & $98.25 \pm 5.990$ & $96.345 \pm 0.799$ \\
\hline ICV-STZ & $15.887 \pm 9.058^{*}$ & $8.625 \pm 8.751^{*}$ & $19.315 \pm 7.751^{\star}$ \\
\hline $\begin{array}{c}\mathrm{STZ}+\mathrm{ChA}(25 \\
\mathrm{mg} / \mathrm{kg} / \mathrm{p} . \mathrm{o})\end{array}$ & $29.715 \pm 6.072 \#$ & $55.75 \pm 4.567 \#$ & $57.810 \pm 7.930 \#$ \\
\hline $\begin{array}{c}\mathrm{STZ}+\mathrm{ChA} \\
(50 \mathrm{mg} / \mathrm{kg} / \mathrm{p} . \mathrm{o})\end{array}$ & 48.875 ×8.392@ & $65.375 \pm 5.930 @$ & 78.346士6.085@ \\
\hline $\begin{array}{c}\mathrm{STZ}+\mathrm{ChA}(100 \\
\mathrm{mg} / \mathrm{kg} / \mathrm{p} . \mathrm{o})\end{array}$ & $52.625 \pm 6.820 @ \#$ & $77.913 \pm 6.085 @ \#$ & $89.625 \pm 7.930 @ \#$ \\
\hline
\end{tabular}

dose dependent improvement $(\mathrm{P}<0.001)$ discriminating abilities of ChA treated rats with that of STZ alone (Figure 3C).

\section{ChA ameliorated hippocampal acetylcholinesterase (AChE) in ICV-STZ- infused rats.}

According to the cholinergic hypothesis, the activity of AChE significantly increases during $\mathrm{AD}$ which leads to degradation of Acetylcholine (ACh). Ventricular administration of STZ produced significant increase in brain AChE activity when compared with that of sham control $(P$ $<0.001)$ (Figure 4). Oral administration of ChA $(100 \mathrm{mg} / \mathrm{kg})$ very significantly attenuated STZ- induced increase in AChE activity $(P<0.001)$. However, ChA (25 and $50 \mathrm{mg} / \mathrm{kg} / \mathrm{p}$.o also showed a decrease in AChE activity in dose dependent manner but with lesser significance when compared with ICV-STZ infused rats.

\section{ChA improve brain catecholamines levels in ICV-STZ infused rats}

STZ $(3 \mu \mathrm{l} / 3 \mathrm{mg} / \mathrm{kg} / \mathrm{ICV})$ treatment caused significant $(p<0.001)$ decrease in levels of catecholamines (NE, DA and 5-HT) in hippocampus 
as compared to sham control group. Treatment with ChA acid (25 and $50 \mathrm{mg} / \mathrm{kg}$ p.o) significantly $(p<0.001)$ improved the levels of NE, DA, 5 -HT as compared to ICV-STZ treated group. At last, ChA $(100 \mathrm{mg} / \mathrm{kg}$ p.o) sdose dependently $(p<0.001)$ attenuated decrease in levels of NE, DA, 5-HT as compared to STZ treated rats (Table 1).

\section{ChA reversed ICV-STZ mediated brain GABA and Glutamate levels in ICV-STZ treated rats}

ICV-STZ $(3 \mu \mathrm{l} / 3 \mathrm{mg} / \mathrm{kg} / \mathrm{ICV})$ treatment produced significant $(p<0.001)$ decrease in levels of GABA and elevated glutamate in hippocampus as compared to normal control group. ChA (50 and $100 \mathrm{mg} / \mathrm{kg}$ p.o) showed more significant $(p<0.001)$ effect by attenuating decrease in levels of GABA and increase in levels of glutamate as compared to ChA (25 mg/ $\mathrm{kg}$ p.o) to STZ treated rats in dose depended manner (Table 2).

\section{ChA treatment prevented ICV-STZ induced rise in} hippocampal pro-inflammatory cytokines (IL-1 $\beta$, IL-6 and TNF- $a$ ) in rats

ICV-STZ $(3 \mathrm{mg} / \mathrm{kg})$ treatment significantly $(p<0.05)$ raised IL-1 $\beta$, IL-6 and TNF- $\alpha$ hippocampal levels as compared to sham control group. This effect is significantly reversed by ChA ( 25 and $50 \mathrm{mg} / \mathrm{kg}$ p.o, $p<0.001$ ) treatment in hippocampus as compared to STZ alone treated group. ChA

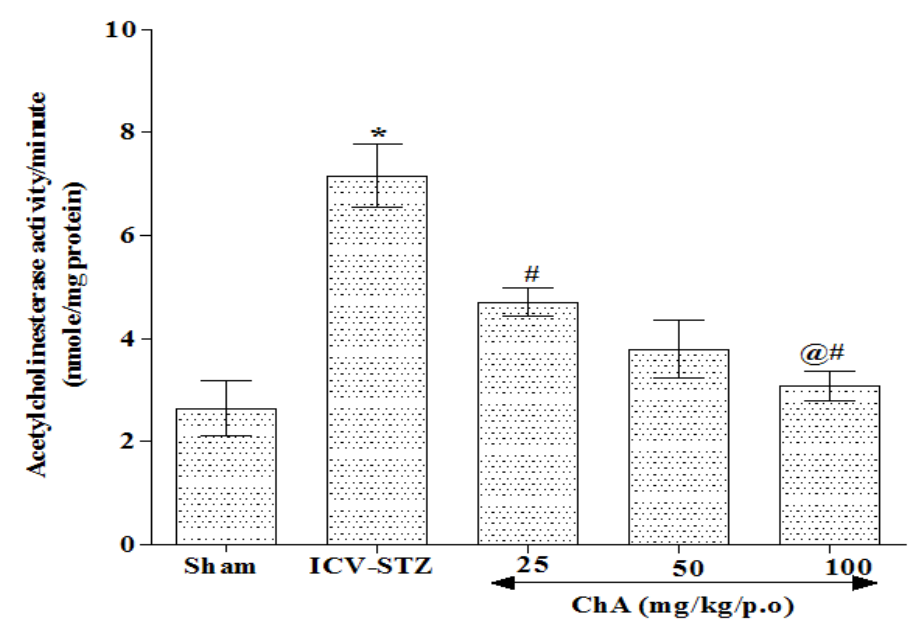

Figure 4: Effect of ChA on brain (hippocampus) Acetylcholinesterase activity in intracerebroventricular streptozotocin (ICV-STZ) treated rats.

Values are expressed as mean $\pm \mathrm{SEM},{ }^{*} \mathrm{P}<0.001$ vs Sham, $P P<0.001$ vs STZ, @ $P<0.001$ vs ChA (25mg/kg), @\# $P<0.001$ vs ChA (50mg/kg).

Table 2: Effect of ChA on brain (hippocampus) GABA and Glutamate in intracerebroventricular streptozocin (ICV-STZ) treated rats.

\begin{tabular}{ccc}
\hline Groups & \multicolumn{2}{c}{ Biochemical Parameters } \\
& ng $/ \mathrm{mg}$ tissue sample (\% of control) \\
& GABA & Glutamate \\
\hline Sham control & $223.625 \pm 1.37$ & $317.625 \pm 0.25$ \\
ICV-STZ & $109.5 \pm 2.44^{*}$ & $478.875 \pm 0.54^{*}$ \\
STZ+ ChA $(25 \mathrm{mg} / \mathrm{kg} / \mathrm{p} .0)$ & $163.625 \pm 2.32 \#$ & $387.85 \pm 0.33 \#$ \\
$\mathrm{STZ}+\mathrm{ChA}(50 \mathrm{mg} / \mathrm{kg} / \mathrm{p} .0)$ & $199.375 \pm 0.75 @$ & $337.874 \pm 0.34 @$ \\
$\mathrm{STZ}+\mathrm{ChA}(100 \mathrm{mg} / \mathrm{kg} / \mathrm{p} . \mathrm{o})$ & $202.375 \pm 1.36 @ \#$ & $323.44 \pm 0.41 @ \#$ \\
\hline
\end{tabular}

Values are expressed as mean $\pm \mathrm{SEM},{ }^{*} P<0.001$ vs Sham, \# $P<0.001$ vs STZ, @ $P<0.001$ vs ChA $(25 \mathrm{mg} / \mathrm{kg})$, @\# $P<0.001$ vs ChA $(50 \mathrm{mg} / \mathrm{kg})$.
$(100 \mathrm{mg} / \mathrm{kg}$ p.o) $(p<0.001)$ showed more marked effects in attenuating the levels of IL-1 $\beta$, IL-6 and TNF- $\alpha$ in hippocampus (Table 3).

\section{ChA reversed ICV-STZ mediated increase in MDA and}

Nitrite level while a decrease in GSH activity in the hippocampus in rats

Increased brain nitrite expression and lipid peroxidation (Malondialdehyde level) and decrease antioxidant (Glutathione level) enzymes which leads to nitrosative and oxidative stress respectively are an integral part of AD-affected brains. Systemic administration of ICV-STZ (3 mg/kg) on day 1 and day 3 following surgery significantly $(p<0.001)$ increased $\mathrm{MDA}$, nitrite concentration and depleted glutathione as compared to sham control group (Table 4). Chronic administration of ChA at all

Table 3: Effect of ChA on brain (hippocampus) proinflammatory markers in intracerebroventricular streptozocin (ICV-STZ) treated rats.

\begin{tabular}{cccc}
\hline Groups & \multicolumn{3}{c}{ Biochemical Parameters (pg/ml) } \\
& TNF-a & IL-1 $\beta$ & II-6 \\
\hline Sham control & $33.31 \pm 6.717$ & $26.10 \pm 5.990$ & $25.17 \pm 0.799$ \\
ICV-STZ & $102.20 \pm 9.058^{*}$ & $89.10 \pm 8.751^{*}$ & $71.15 \pm 7.751^{*}$ \\
$\begin{array}{c}\text { STZ+ ChA (25 mg/ } \\
\text { kg/ p.o })\end{array}$ & $88.11 \pm 6.072 \#$ & $72.16 \pm 4 \#$ & $66.80 \pm 7.930 \#$ \\
$\begin{array}{c}\text { STZ+ ChA (50mg/ } \\
\text { kg/ p.o) }\end{array}$ & $74.41 \pm 8.392 @$ & $67.96 \pm 5.930 @$ & $59.46 \pm 6.085 @$ \\
$\begin{array}{c}\text { STZ+ ChA (100 } \\
\text { mg/kg/p.o) }\end{array}$ & $68.79 \pm 6.820 @ \#$ & $41.93 \pm 6.085 @ \#$ & $37.80 \pm 7.930 @ \#$ \\
\hline
\end{tabular}

Values are expressed as mean $\pm \mathrm{SEM},{ }^{*} P<0.001$ vs Sham, $\# P<0.001$ vs STZ, @ $P<0.001$ vs ChA $(25 \mathrm{mg} / \mathrm{kg})$, @\# $P<0.001$ vs ChA $(50 \mathrm{mg} / \mathrm{kg})$

Table 4: Effect of ChA on brain (hippocampus) biochemical parameters and on spontaneous locomotor activity in intracerebroventricular streptozocin (ICV-STZ) treated rats.

\begin{tabular}{|c|c|c|c|c|}
\hline \multirow[t]{2}{*}{ Groups } & \multicolumn{3}{|c|}{ Biochemical Parameters } & \multirow{2}{*}{$\begin{array}{l}\text { Locomotor } \\
\text { activity } \\
\text { Activity } \\
\text { counts/10 } \\
\text { minutes }\end{array}$} \\
\hline & $\begin{array}{c}\text { MDA } \\
\text { (nmole/mg } \\
\text { protein) }\end{array}$ & $\begin{array}{c}\text { Nitrite } \\
\text { ( } \mu \text { mole/mg } \\
\text { protein) }\end{array}$ & $\begin{array}{c}\text { GSH } \\
\text { ( } \mu \text { mole/mg } \\
\text { protein) }\end{array}$ & \\
\hline $\begin{array}{l}\text { Sham } \\
\text { control }\end{array}$ & $0.365 \pm 1.37$ & $7.72 \pm 0.25$ & $2.129 \pm 0.23$ & $221.66 \pm 10.99$ \\
\hline ICV-STZ & $1.933 \pm 2.44$ * & $26.5 \pm 0.54$ * & $0.029 \pm 0.08$ * & $205.66 \pm 11.77$ \\
\hline $\begin{array}{c}\mathrm{ChA}+ \\
\mathrm{STZ} \\
(25 \mathrm{mg} / \\
\mathrm{kg} / \mathrm{p} . \mathrm{o})\end{array}$ & $0.840 \pm 2.32 \#$ & $11.85 \pm 0.33 \#$ & $0.183 \pm 0.18 \#$ & $207.83 \pm 12.45$ \\
\hline $\begin{array}{c}\mathrm{ChA}+ \\
\mathrm{STZ} \\
(50 \mathrm{mg} / \\
\mathrm{kg} / \mathrm{p} . \mathrm{o})\end{array}$ & $0.735 \pm 0.75 @$ & $9.84 \pm 0.34 @$ & 1.079土0.88@ & $\begin{array}{c}228.167 \pm \\
11.83\end{array}$ \\
\hline $\begin{array}{c}\mathrm{ChA}+ \\
\mathrm{STZ} \\
(100 \mathrm{mg} / \\
\mathrm{kg} / \mathrm{p} . \mathrm{o})\end{array}$ & $\begin{array}{l}0.636 \pm \\
1.36 @ \#\end{array}$ & $\begin{array}{c}8.44 \pm \\
0.41 @ \#\end{array}$ & $1.156 \pm 0.28 @ \#$ & $202.167 \pm 9.43$ \\
\hline
\end{tabular}

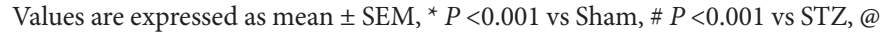
$P<0.001$ vs ChA $(25 \mathrm{mg} / \mathrm{kg})$, @\# $P<0.001$ vs ChA $(50 \mathrm{mg} / \mathrm{kg})$

Note: Malondialdehyde (MDA), reduced glutathione (GSH) 
three doses attenuated STZ induced elevation in MDA, nitrite levels and restored levels of antioxidant enzyme glutathione as compared to STZ infused group.

\section{DISCUSSION}

Despite remarkable progress in understanding pathogenesis of dementia, the search for a cure against these diseases is troublesome and frustrating; for Decades now, complications of the $\mathrm{AD}$ have made the development of its therapeutic intervention quite a challenging task. ${ }^{29}$ Although the currently prescribed molecules provide some improvement in the clinical condition of patients, it is at a cost of having to bear the burden of their adverse effects and lack of their curative effects. ${ }^{37}$ Thus it is imperative to keep exploring different approaches that can be used to target the $\mathrm{AD}$ complications which could be translated into successful clinical trials. The herbs have been used as medicines from ancient times, as they are safer and have no side effects. The ultimate aim of this research is to find a solution for this disease by using herbal compound. In present study we demonstrate the therapeutic potential of ChA in ICV-STZ induced experimental sporadic AD. ICV-STZ induced model has been commonly used to explore the various behavioral, biochemical and cellular alterations, implicated in pathogenesis of SAD. In the present study, bilateral ICV infusion of STZ produced cognitive impairment, cholinergic deficiency, elevation in proinflammatory cytokines, oxidative stress and hippocampus neurochemical and histopathologicsal alterations in rats..$^{38}$ The observed changes are in line with earlier studies demonstrating similar behavioral and biochemical alterations following STZ infusion in rats. ICV administration of STZ has been reported to produce $\mathrm{AD}$ like symptoms in animals and non-human primates. The findings of the present study are in tune with earlier reports which also observed behavioral, biochemical, neurochemical and histopathologicsal changes in rats following ICV-STZ infusion in rats. In the present study results of memory consolidation and novel object recognition in ICV-STZ infusion in rats, evaluated by MWM and OR paradigms shown poor learning and discriminative abilities. Although, the exact mechanism of ICV-STZ induced cognitive deficits is not clear. However, various mechanisms such as cellular energy failure, mitochondrial damage, oxidative stress, excitotoxicity, upregulation of inflammatory markers, degeneration of cholinergic neurons and ultimately leading to cell death which are primarily linked with the deterioration of learning and memory abilities has been correlated in STZ induced learning and memory impairment. ${ }^{38}$ The present findings illustrated the ICV-STZ infused rats significantly prolonged escape latency in MWM task as compared to animals of sham control, suggesting ICV-STZ impaired spatial learning and memory. Similar observations were also observed in novel ORT that define spatial learning, origination of new memories and recapitulations of stored memories. ${ }^{39}$ In the present study, ICV-STZ administrated rats were unable to discriminate between familial and novel objects in ORT. The changes in spontaneous locomotor activity have been suggested to modulate the learning and memory in ORT and MWM paradigms. ${ }^{28,40}$ However, no significant difference in spontaneous locomotor activity was observed in any of the experimental group. ChA treated rats showed dose dependently significant improvement in acquisition and consolidation and were able to discriminate between familiar and novel objects suggesting improvement of learning and memory in STZ treated rats without affecting spontaneous locomotor activity in line with the previous reports. ${ }^{41}$ The results of current study show that ChA displayed behavioral profile that is consistent with an antidepressant and anxiolytic actions (Onasanwo et al. 2014) and neuroprotective potential glutamate induced excitotoxicity. In line with previous observations, the behavioral disruption in STZ treated rats observed in present study may be due to upregulation of $\mathrm{AChE}$ enzyme ${ }^{28,41}$ further supporting the impairment of cholinergic system viz loss of memory function and coordination. It has been reported that Terminelia chebula posses AchE inhibitory activity. ${ }^{18}$ In the present study, administration of CA significantly attenuated the elevated levels of AChE whereas these effects were more profound when CA was given at higher doses. Evidently the improved memory performance was observed in MWM and novel ORT as well as improvement of AChE enzyme level in STZ treated group by administration of CA suggesting its potential role in cognitive performance.

It has been demonstrated that hippocampus is highly enriched in cholinergic, glutamenrgic, GABAergic and monoaminergic axon terminals and these neurotransmitters known to play crucial role in encoding, storage and expression of memory. ${ }^{42} \mathrm{~A}$ close relationship exists between impairment in behavioral function and disruption of neurotransmitters homeostasis in hippocampus in $\mathrm{AD}$ as well as in experimental animals. ${ }^{43,44}$ Linked with this, these alterations in hippocampal neurochemistry following ICV-STZ infusion in rats in the present study. On the other hand, significant alterations in hippocampal neurochemistry including deficits in monoamines (NE, DA and 5HT) and disturbed balance of GABA and Glutamate have also been reported to occur in $\mathrm{AD}^{45,46}$ as well as following ICV-STZ infusion in rats. ${ }^{41}$ In the present study, ICV-STZ treatment significantly decreases GABA, DA, NE, 5-HT and increases glutamate level. In the present study, treatment with $\mathrm{ChA}$ significantly restored the hippocampal neurotransmitters signaling implicated that ChA may improve neurotransmitters homeostasis in hippocampus in STZ treated animals and also in pathophysiology of AD.Oxidative stress is initiated by reactive oxygen species (ROS), which are produced as a by-product of electron transport in mitochondria play a key pathogenic role in disease progression and thought to be involved in STZ induced cognitive deterioration. ${ }^{47}$ Previous findings suggested that mitochondria consist of multiple electron carriers competent of producing ROS and widespread network of antioxidant defence mechanism. Any abnormality or internal insult to mitochondrial can cause an imbalance between generation of ROS and defense, leading to oxidative damage. ${ }^{8}$ Interestingly, ROS not only cause damage to cellular structures which lead to neurotic cell death, but also provoke cellular responses which are evident in vulnerable neurons in AD. Excess ROS causes cell injury by damaging lipids, proteins and DNA in cell. ${ }^{48}$ In the present study, ICV administration of STZ significantly increased MDA and nitrite concentration, depleted the levels of reduced glutathione and SOD, signifying oxidative damage. Recent studies showed the antioxidant properties of different extracts of $T$. chebula fruits. ${ }^{10}$ In an earlier report, a $70 \%$ methanol extract of T. chebula fruits was found to have good efficacy in radical scavenging abilities. ${ }^{25} \mathrm{In}$ another report, chloroform, ethanolic, n-butanolic and organic aqueous extracts were investigated for anti-lipidperoxidation, anti-superoxide radical formation and free radical scavenging activities. ${ }^{10}$ Thus in the present study, chronic administration of ChA signifanctly attenuated oxidative damage, by attenuating MDA and nirite level and improve antioxidant defence, demonstrating its anti-oxidative effect. Moreover, this antioxidant effect was enhanced when $\mathrm{ChA}$ is given at higher dose.

Neuroinflammation also plays major role in pathogenesis of CNS disorders including $\mathrm{AD} .{ }^{49}$ Several hypotheses support the fact that oxidative damage and mitochondrial dysfunctions are the major contributors of neuroinflammation. ${ }^{50}$ Cytokines such as IL- 1 , IL- $\beta$ and TNF- $\alpha$ has been implicated in the pathogenesis of various neurodegenerative diseases. ROS generation by IL-1, IL- $\beta$ and TNF- $\alpha$ leads to the increased expression of various inflammatory genes like MMP-9 which may increase $\mathrm{BBB}$ permeability, causing recruitment of immune cells infiltrating through $\mathrm{BBB}$ into tissues and subsequently results in neuroinflammation..$^{51}$ Moreover, it has been reported that IL- $1 \beta$ and IL- 6 induces neurotoxicity mainly through the release of free radicals. In addition in brain microglia produces TNF- $\alpha$ and its overproduction has been linked 
with neurodegeneration. ${ }^{52}$ These studies indicate that cytokines such as IL-1 $\beta$, IL- 6 and TNF- $\alpha$, contributes to the CNS inflammation and neurodegeneration. In the present study, ICV- STZ infusion caused significant elevation in the hippocampus cytokines levels indicating neuroinflammation and this stz induced elevation in proinflammatory cytokines was significantly attenuated by ChA treatment.

\section{CONCLUSION}

Therefore, we predict that, ChA dose dependently attenuated STZ- induced cognitive deterioration and other biochemical and neurochemical alterations in the present study. ChA produced neuroprotective action by reduction of nitro-oxidative stress and anti-inflammatory activities and its ability to modulate hippocampal neurochemistry and neuronal cell death. The current study further provides a hope that these ChA could be used in the treatment and management of cognitive disorders such as AD. However, their exact mechanism at cellular and molecular level is still poorly understood and needs to be explored further.

\section{ACKNOWLEDGEMENT}

Authors are thankful to Mr. Parveen Garg, the Chairman, ISF College of Pharmacy, Moga, (Punjab) for his praiseworthy inspiration and support for this study. Authors are also grateful and sincerely acknowledge IKGPTU, Kapurthala (Punjab), for expediting the research proposal and improving the research outcomes by facilitating interactions with experts.

\section{CONFLICT OF INTEREST}

The authors declare no conflict of interest.

\section{REFERENCES}

1. Morita Y, Shibutani T, Nakanishi N, Nishikura K, Iwai S, Kuraoka I. Human endonuclease $\mathrm{V}$ is a ribonuclease specific for inosine-containing RNA. Nat Commun. 2013;4(1):2273.

2. Bature F, Guinn BA, Pang D, Pappas Y. Signs and symptoms preceding the diagnosis of Alzheimer's disease: A systematic scoping review of literature from 1937 to 2016. BMJ Open. 2017;7(8):e015746.

3. Patterson C. World Alzheimer Report 2018: The state of the art of dementia research: New frontiers. Alzheimer's Dis Int London, UK. 2018;1-48.

4. Du X, Wang X, Geng M. Alzheimer's disease hypothesis and related therapies. Transl Neurodegener. 2018;7(1):1-7.

5. Gonçalves FM, Neis VB, Rieger DK, Lopes MW, Heinrich IA, Costa AP, et al. Signaling pathways underlying the antidepressant-like effect of inosine in mice. Purinergic Signal. 2016. Available from: http://dx.doi.org/10.1007/s11302-0169551-2

6. Rahman A. The Role of Adenosine in Alzheimer 's disease. Current Neuropharmacology. 2009;7(3):207-16.

7. Zhou S, Yu G, Chi L, Zhu J, Zhang W, Zhang Y, et al. Neuroprotective effects of edaravone on cognitive deficit, oxidative stress and tau hyperphosphorylation induced by intracerebroventricular streptozotocin in rats. Neurotoxicology. 2013;38:136-45. Available from: http://dx.doi.org/10.1016/j.neuro.2013.07.007

8. Sharma V, Bala A, Deshmukh R, Bedi KL, Sharma PL. Neuroprotective effect of RO-20-1724-a phosphodiesterase4 inhibitor against intracerebroventricular streptozotocin induced cognitive deficit and oxidative stress in rats. Pharmacol Biochem Behav. 2012;101(2):239-45.

9. Gutierres JM, Carvalho FB, Schetinger MRC, Marisco P, Agostinho P, Rodrigues $M$, et al. Anthocyanins restore behavioral and biochemical changes caused by streptozotocin-induced sporadic dementia of Alzheimer's type. Life Sci. 2014;96(1-2):7-17.

10. Saha S, Verma RJ. Antioxidant activity of polyphenolic extract of Terminalia chebula Retzius fruits. J Taibah Univ Sci. 2016;10(6):805-12.

11. ElHalawany AM, Sayed NSEL, Abdallah HM, EIDine RS. Protective effects of gingerol on streptozotocin-induced sporadic Alzheimer's disease: Emphasis on inhibition of $\beta$-amyloid, COX-2, alpha-, beta-secretases and APH1a. Sci Rep. $2017 ; 7(1): 1-11$.

12. Onasanwo S, Faborode SO, Agrawal M, ljiwola OL, Jaiyesimi BO, Narender T. Antidepressant and anxiolytic potentials of chebulinic acid in laboratory rodent. Ann Depress Anxiety. 2014;1(7):1032.

13. Pfundstein B, EIDesouky SK, Hull WE, Haubner R, Erben G, Owen RW. Polyphenolic compounds in the fruits of Egyptian medicinal plants (Terminalia bel-
Ierica, Terminalia chebula and Terminalia horrida): Characterization, quantitation and determination of antioxidant capacities. Phytochemistry. 2010;71(10):113248.

14. Nampoothiri SV, Prathapan A, Cherian OL, Raghu KG, Venugopalan VV, Sundaresan A. In vitro antioxidant and inhibitory potential of Terminalia bellerica and Emblica officinalis fruits against LDL oxidation and key enzymes linked to type 2 diabetes. Food Chem Toxicol. 2011;49(1):125-31.

15. Cho CH, Kim EA, Kim J, Choi SY, Yang SJ, Cho SW. N-Adamantyl-4-methylthiazol-2-amine suppresses amyloid $\beta$-induced neuronal oxidative damage in cortical neurons. Free Radic Res. 2016;50(6):678-90.

16. Lee HS, Jung SH, Yun BS, Lee KW. Isolation of chebulic acid from Terminalia chebula Retz. and its antioxidant effect in isolated rat hepatocytes. Arch Toxicol. 2007;81(3):211-8.

17. Nair V, Singh S, Gupta YK. Anti-arthritic and disease modifying activity of Terminalia chebula Retz. in experimental models. J Pharm Pharmacol. 2010;62(12):1801-6.

18. Nag G, De B. Acetylcholinesterase inhibitory activity of Terminalia chebula, Terminalia bellerica and Emblica officinalis and some phenolic compounds. Int J Pharm Pharm Sci. 2011;3(3):121-4.

19. Chang CL, Lin CS. Phytochemical composition, antioxidant activity and neuroprotective effect of Terminalia chebula Retzius extracts. Evidence-Based Complement Altern Med. 2012;2012.

20. Chen S-Y, Zheng K, Wang Z. Neuroprotective effects of ellagic acid on neonatal hypoxic brain injury via inhibition of inflammatory mediators and down-regulation of JNK/p38 MAPK activation. Trop J Pharm Res. 2016;15(2):241-51.

21. Yang MH, Vasquez Y, Ali Z, Khan IA, Khan SI. Constituents from Terminalia species increase PPAR $\alpha$ and PPAR $\gamma$ levels and stimulate glucose uptake without enhancing adipocyte differentiation. J Ethnopharmacol. 2013;149(2):490-8.

22. Saleem A, Husheem M, Härkönen P, Pihlaja K. Inhibition of cancer cell growth by crude extract and the phenolics of Terminalia chebula Retz. fruit. J Ethnopharmacol. 2002;81(3):327-36.

23. Lee Y, Byun HS, Seok JH, Park KA, Won M, Seo W, et al. Terminalia chebula provides protection against dual modes of necroptotic and apoptotic cell death upon death receptor ligation. Sci Rep. 2016;6:25094.

24. Silawat N, Gupta VB. Chebulic acid attenuates ischemia reperfusion induced biochemical alteration in diabetic rats. Pharm Biol. 2013;51(1):23-9.

25. Hazra B, Sarkar R, Biswas S, Mandal N. Comparative study of the antioxidant and reactive oxygen species scavenging properties in the extracts of the fruits of Terminalia chebula, Terminalia belerica and Emblica officinalis. BMC Complement Altern Med. 2010;10(1):20.

26. Jing $Y H$, Gao LP, Ren YX, Yin J, Wang JQ, Zhang L, et al. Brain Aging and AD-Like Pathology in Streptozotocin-Induced Diabetic Rats. J Diabetes Res. 2014;2014:1-12.

27. Paxinos G, Watson C. The rat brain in stereotaxic coordinates. gth edn. San Diego. Academic Press. 1999.

28. Deshmukh R, Sharma V, Mehan S, Sharma N, Bedi KL. Amelioration of intracerebroventricular streptozotocin induced cognitive dysfunction and oxidative stress by vinpocetine: A PDE1 inhibitor. Eur J Pharmacol. 2009;620(1-3):49-56.

29. Arora R, Deshmukh R. Embelin attenuates intracerebroventricular streptozotocin-induced behavioral, biochemical and neurochemical abnormalities in rats. Mol Neurobiol. 2017;54(9):6670-80.

30. Giorgetti M, Gibbons JA, Bernales S, Alfaro IE, LaRochelle CD, Cremers T, et al. Cognition-enhancing properties of Dimebon in a rat novel object recognition task are unlikely to be associated with acetylcholinesterase inhibition or N-methyl-D-aspartate receptor antagonism. J Pharmacol Exp Ther. 2010;333(3):748-57.

31. Lowry OH, Rosebrough NJ, Farr AL, Randall RJ. Protein measurement with the Folin phenol reagent. J Biol Chem. 1951;193(1):265-75.

32. Ellman GL, Courtney KD, JrAndres V, Featherstone RM. A new and rapid colorimetric determination of acetylcholinesterase activity. Biochem Pharmacol. $1961 ; 7(2): 88-95$

33. Wills E. Mechanisms of lipid peroxide formation in animal tissues. Biochem J. 1966:99(3):667.

34. Ellman GL. Tissue sulfhydryl groups. Arch Biochem Biophys. 1959;82(1):70-7.

35. Green LC, Wagner DA, Glogowski J, Skipper PL, Wishnok JS, Tannenbaum SR Analysis of nitrate, nitrite and [15N] nitrate in biological fluids. Anal Biochem. 1982;126(1):131-8.

36. Donzanti BA, Yamamoto BK. An improved and rapid HPLC-EC method for the isocratic separation of amino acid neurotransmitters from brain tissue and microdialysis perfusates. Life Sci. 1988;43(11):913-22.

37. Kumar A, Sharma V, Singh VP, Kaundal M, Gupta MK, Bariwal J, et al. Herbs to curb cyclic nucleotide phosphodiesterase and their potential role in Alzheimer's disease. Mech Ageing Dev. 2015;149:75-87.

38. Salkovic-Petrisic M, Osmanovic-Barilar J, Knezovic A, Hoyer S, Mosetter K, Reutter W. Long-term oral galactose treatment prevents cognitive deficits in male Wistar rats treated intracerebroventricularly with streptozotocin. Neuropharmacology. 2014;77:68-80.

39. Mehla J, Pahuja M, Gupta YK. Streptozotocin-induced sporadic Alzheimer 's disease: Selection of appropriate dose. J Alzheimer's Dis. 2013;33(1):17-21. 
40. Rani V, Deshmukh R, Jaswal P, Kumar P, Bariwal J. Alzheimer's disease: Is this a brain specific diabetic condition?. Physiol Behav. 2016;164(Pt A):259-67. Available from: http://dx.doi.org/10.1016/j.physbeh.2016.05.041

41. Arora R, Deshmukh R. Embelin attenuates Intracerebroventricular Streptozotocin-Induced Behavioral, Biochemical and Neurochemical Abnormalities in Rats. Mol Neurobiol. 2016. Available from: http://dx.doi.org/10.1007/s12035016-0182-y

42. Dani JA, Bertrand D. Nicotinic Acetylcholine Receptors and Nicotinic Cholinergic Mechanisms of the Central Nervous System. 2007;47:699-729.

43. Lu F, Li X, Li W, Wei K, Yao Y, Zhang Q, et al. Tetramethylpyrazine reverses intracerebroventricular streptozotocin-induced memory deficits by inhibiting GSK3ß. Acta Biochim Biophys Sin. 2017;49(8):722-8.

44. Sun P, Ortega G, Tan Y, Hua Q, Riederer PF, Deckert J, et al. Streptozotocin impairs proliferation and differentiation of adult hippocampal neural stem cells in vitro-correlation with alterations in the expression of proteins associated with the insulin system. Front Aging Neurosci. 2018;10:145

45. Chen Y, Liang Z, Blanchard J, Dai CL, Sun S, Lee MH, et al. A non-transgenic mouse model (icv-STZ mouse) of Alzheimer's disease: similarities to and differences from the transgenic model ( $3 \times T$ Tg-AD mouse). Mol Neurobiol. 2013;47(2):711-25.
46. Wang R, Reddy PH. Role of glutamate and NMDA receptors in Alzheimer's disease. J Alzheimer's Dis. 2017;57(4):1041-8.

47. Grieb P. Intracerebroventricular Streptozotocin Injections as a Model of Alzheimer's Disease: In Search of a Relevant Mechanism. Mol Neurobiol. 2016;53(3):1741-52.

48. Federico A, Cardaioli E, DaPozzo P, Formichi P, Gallus GN, Radi E. Mitochondria oxidative stress and neurodegeneration. J Neurol Sci. 2012;322(1-2):254-62.

49. Stamouli EC, Politis AM. Pro-inflammatory cytokines in Alzheimer's disease. Psychiatr. 2016;27(4):264-75.

50. Montgomery SL, Bowers WJ. Tumor necrosis factor-alpha and the roles it plays in homeostatic and degenerative processes within the central nervous system. J Neuroimmune Pharmacol. 2012;7(1):42-59.

51. Calabrese V, Cornelius C, Leso V, Trovato-Salinaro A, Ventimiglia B, Cavallaro M, et al. Oxidative stress, glutathione status, sirtuin and cellular stress response in type 2 diabetes. Biochim Biophys Acta: Mol Basis Dis. 2012;1822(5):729-36. Available from: http://dx.doi.org/10.1016/j.bbadis.2011.12.003

52. Paouri E, Tzara O, Kartalou GI, Zenelak S, Georgopoulos S. Peripheral tumor necrosis factor-alpha (TNF- $\alpha$ ) modulates amyloid pathology by regulating bloodderived immune cells and glial response in the brain of AD/TNF transgenic mice. J Neurosci. 2017;37(20):5155-71.

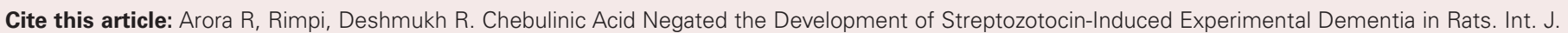
Pharm. Investigation. 2020;10(1):24-31. 